\title{
COMPUTING POLYNOMIAL SOLUTIONS OF EQUIVARIANT POLYNOMIAL ABEL DIFFERENTIAL EQUATIONS
}

\author{
JAUME LLIBRE ${ }^{2}$ AND CLÀUDIA VALLS ${ }^{3}$
}

\begin{abstract}
Let $a(x)$ non-constant and $b_{j}(x)$ for $j=0,1,2,3$ be real or complex polynomials in the variable $x$. Then the real or complex equivariant polynomial Abel differential equations $a(x) \dot{y}=b_{1}(x) y+$ $b_{3}(x) y^{3}$ with $b_{3}(x) \neq 0$, and the real or complex polynomial equivariant polynomial Abel differential equations of second kind $a(x) y \dot{y}=b_{0}(x)+$ $b_{2}(x) y^{2}$ with $b_{2}(x) \neq 0$, have at most 7 polynomial solutions. Moreover there are equations of these type having these maximum number of polynomial solutions.
\end{abstract}

\section{Introduction AND Statement of the main RESUlts}

Abel differential equations of first kind

$$
a(x) \dot{y}=b_{0}(x)+b_{1}(x) y+b_{2}(x) y^{2}+b_{3}(x) y^{3}
$$

with $b_{3}(x) \neq 0$ appear in many text-books of ordinary differential equations as one of first non-trivial examples of nonlinear differential equations, see for instance [10]. Here the dot denotes the derivative with respect to the independent variable $x$. If $b_{3}(x)=b_{0}(x)=0$ or $b_{2}(x)=b_{0}(x)=0$ the Abel differential equation reduces to a Bernoulli differential equation, while if $b_{3}(x)=0$ the Abel differential equation reduces to a Riccati differential equation.

The Abel differential equations (1) have been studied intensively, either calculating their solutions (see for instance $[7,11,12,13]$ ), or classifying their centers (see $[2,3,4]$ ), and recently in $[6,8,9]$ the authors studied the polynomial solutions of the differential equation $y^{\prime}=\sum_{i=0}^{n} a_{i}(x) y^{i}$.

The analysis of particular solutions (as polynomial or rational solutions) of the differential equations is important for understanding the set of solutions of a differential equation. In 1936 Rainville [14] characterized the Riccati differential equations $\dot{y}=b_{0}(x)+b_{1}(x) y+y^{2}$, with $b_{0}(x)$ and $b_{1}(x)$ polynomials in the variable $x$, having polynomial solutions.

2010 Mathematics Subject Classification. Primary 34A05. Secondary 34C05, 37C10. Key words and phrases. polynomial Abel equations, equivariant polynomial equation, polynomial solutions. 
Campbell and Golomb [5] in 1954 provides an algorithm for determining the polynomial solutions of the Riccati differential equation $a(x) y^{\prime}=b_{0}(x)+$ $b_{1}(x) y+b_{2}(x) y^{2}$, where $a, b_{0}, b_{1}, b_{2}$ are polynomials in the variable $x$. Behloul and Cheng [1] in 2006 gave a different algorithm for finding the rational solutions of the differential equations $a(x) y^{\prime}=\sum_{i=0}^{n} b_{i}(x) y^{i}$, where $a, b_{i}$ are polynomials in the variable $x$.

Here we consider the Abel differential equations (1) where $a(x) \in \mathbb{F}[x] \backslash$ $\{0\}, b_{i}(x) \in \mathbb{F}[x], i=0,1,2,3, b_{3}(x) \neq 0$, where $\mathbb{F}=\mathbb{R}, \mathbb{C}$, and $\mathbb{F}[x]$ is the ring of polynomials in the variable $x$ with coefficients in $\mathbb{F}$. We also assume that $a(x)$ is not constant. The case $a(x)$ constant has been studied in [9]. We say that the Abel differential equation (1) has degree $\eta$.

Equation (1) is reversible with respect to the change of variables $(x, y) \rightarrow$ $(x,-y)$ if the following equation

$$
-a(x) \dot{y}=-\left(b_{0}(x)-b_{1}(x) y+b_{2}(x) y^{2}-b_{3}(x) y^{3}\right)
$$

coincides with equation (1). In particular this implies $b_{1}(x)=b_{3}(x)=0$, and since $b_{3}(x)=0$ we do not consider these reversible differential equations.

The Abel differential equation (1) is equivariant with respect to the change of variables $(x, y) \rightarrow(x,-y)$ if the following equation

$$
-a(x) \dot{y}=b_{0}(x)-b_{1}(x) y+b_{2}(x) y^{2}-b_{3}(x) y^{3}
$$

coincides with equation (1). This implies $b_{0}(x)=b_{2}(x)=0$. In this paper first we focus our study in these kind of equivariant polynomial Abel equations, i.e. in the equations

$$
a(x) \dot{y}=b_{1}(x) y+b_{3}(x) y^{3} .
$$

Theorem 1. Real or complex equivariant polynomial Abel differential equations with $b_{3}(x) \neq 0$ and $a(x)$ non-constant, have at most 7 polynomial solutions. Moreover there are equations of this type having these maximum number of polynomial solutions.

The proof of Theorem 1 is given in section 2 .

Our second objective in this paper is on the Abel differential equations of second kind, i.e. on the equations of the form

$$
a(x) y \dot{y}=b_{0}(x)+b_{1}(x) y+b_{2}(x) y^{2},
$$

where $a(x), b_{i}(x) \in \mathbb{F}[x]$ for $i=0,1,2$, with $a(x)$ and $b_{2}(x)$ non-zero. We also consider the ones that are equivariant with respect to the change $(x, y) \rightarrow$ $(x,-y)$. Then we have that $b_{1}(x)=0$ and so equation (3) becomes

$$
a(x) y \dot{y}=b_{0}(x)+b_{2}(x) y^{2} .
$$

We also assume that $a(x)$ is not constant, because the case $a(x)$ constant has been studied in [6]. We say that the equivariant polynomial Abel differential equation of second kind (4). 
Theorem 2. Real or complex equivariant polynomial Abel differential equations of second kind with $b_{2}(x) \neq 0$ and $a(x)$ non-constant, have at most 7 polynomial solutions. Moreover there are equations of this type having these maximum number of polynomial solutions.

The proof of Theorem 2 is given in section 3 .

\section{Proof of Theorem 1}

First we recall that if $y(x) \neq 0$ is a solution of equation (2), then $-y(x)$ is also a solution of equation (2) which is different from $y(x)$.

Lemma 3. Let $y_{0}(x) \neq 0, y_{1}(x), y_{2}(x)$ be polynomial solutions of equation (2) such that $y_{1}(x) \not \equiv 0, y_{2}(x) \not \equiv 0$ and $y_{2}(x) \neq-y_{1}(x)$. Set $y_{1}(x)=$ $g(x) \tilde{y}_{1}(x)$ and $y_{2}(x)=g(x) \tilde{y}_{2}(x)$ where $g=\operatorname{gcd}\left(y_{1}, y_{2}\right)$. Then, except the solution $y=0$, all the other polynomial solutions of equation (2) can be expressed as

$$
y_{0}(x ; c)= \pm \frac{\tilde{y}_{1}(x) \tilde{y}_{2}(x) g(x)}{\left(c \tilde{y}_{1}^{2}(x)+(1-c) \tilde{y}_{2}^{2}(x)\right)^{1 / 2}},
$$

where $c$ is a constant and $\left(c \tilde{y}_{1}^{2}(x)+(1-c) \tilde{y}_{2}^{2}(x)\right)^{1 / 2}$ is a polynomial.

Proof. Let $y$ be a nonzero polynomial solution of equation (2). The functions $z_{0}=1 / y_{0}^{2}, z_{1}=1 / y_{1}^{2}$ and $z_{2}=1 / y_{2}^{2}$ are solutions of a linear differential equation and satisfy

$$
-a(x) \dot{z}_{i}=2 b_{1}(x) z_{i}+2 b_{3}(x), \quad i=0,1,2 .
$$

Therefore we have

$$
\frac{\dot{z}_{0}(x)-\dot{z}_{1}(x)}{z_{0}(x)-z_{1}(x)}=\frac{\dot{z}_{2}(x)-\dot{z}_{1}(x)}{z_{2}(x)-z_{1}(x)} .
$$

Integrating this equality we obtain

$$
z_{0}(x)=z_{1}(x)+c\left(z_{2}(x)-z_{1}(x)\right),
$$

with $c$ an arbitrary constant. So the general solution of equation (2) is

$$
\begin{aligned}
y_{0}^{2}(x) & =\frac{1}{z_{0}(x)}=\frac{1}{z_{1}(x)+c\left(z_{2}(x)-z_{1}(x)\right)} \\
& =\frac{y_{1}^{2}(x) y_{2}^{2}(x)}{c y_{1}^{2}(x)+(1-c) y_{2}^{2}(x)}=\frac{\tilde{y}_{1}^{2}(x) \tilde{y}_{2}^{2}(x) g(x)^{2}}{c \tilde{y}_{1}^{2}(x)+(1-c) \tilde{y}_{2}^{2}(x)},
\end{aligned}
$$

with $c$ an arbitrary constant.

In view of Lemma 3 , if $y_{1}(x), y_{2}(x)$ are polynomial solutions of equation (2) such that $y_{1}(x) \not \equiv 0, y_{2}(x) \not \equiv 0, y_{2}(x) \neq-y_{1}(x)$, then any other polynomial solution different from them is of the form given in (5) for some appropriate constant $c$ such that $c \notin\{0,1\}$. In particular, $c \tilde{y}_{1}^{2}(x)+(1-c) \tilde{y}_{2}^{2}(x)$ is a square of a polynomial $P$ and $P$ divides $g$. We claim that this $c$ is unique. 
We write the condition that $c \tilde{y}_{1}^{2}(x)+(1-c) \tilde{y}_{2}^{2}(x)$ is a square of a polynomial in the form

$$
\tilde{y}_{1}^{2}+d \tilde{y}_{2}^{2}=Z_{1}^{2}
$$

where $d=(1-c) / c$ (we recall that $c \notin\{0,1\})$. We claim that there is a unique $d$ for which this is possible.

For proving the claim we proceed by contradiction. Assume that there exists $d_{1}, d_{2}$ for which

$$
\tilde{y}_{1}^{2}+d_{1} \tilde{y}_{2}^{2}=Z_{1}^{2} \quad \text { and } \quad \tilde{y}_{1}^{2}+d_{2} \tilde{y}_{2}^{2}=Z_{2}^{2},
$$

for some polynomials $Z_{1}, Z_{2}$ and $d_{1}, d_{2} \neq 1$ with $d_{1} \neq d_{2}$. First we state and prove an auxiliary result.

Lemma 4. The polynomial solutions of $X^{2}+Y^{2}=Z^{2}$ with pairwise coprime polynomials $X, Y, Z$ are of the form

$$
\pm X=2 a b, \quad \pm Y=a^{2}-b^{2}, \quad \pm Z=a^{2}+b^{2}
$$

(or with $X, Y$ interchanged) where $a$ and $b$ are co-prime polynomials.

Proof. It is sufficient to consider the plus case because if $X, Y, Z$ is a solution so are $\pm X, \pm Y, \pm Z$. We can assume that are pairwise co-prime as, if a polynomial divides two of them it must divide the third and can be canceled from the identity. Let $X=2 u, Y+Z=2 v$ and $Y-Z=2 w$ with $u, v, w$ polynomials and where $v$ and $w$ are coprime. It follows from the relation

$$
X^{2}=Z^{2}-Y^{2}=(Z+Y)(Z-Y)
$$

that

$$
u^{2}=v w .
$$

So $v, w$ must be squares as they are co-prime. Let $v=a^{2}$ and $w=b^{2}$ where $a$ and $b$ are coprime. Hence

$$
Z=a^{2}+b^{2}, \quad Y=a^{2}-b^{2} \quad X=2 a b
$$

and the lemma is proved.

In the first identity in (6) using Lemma 4 we can write

$$
\tilde{y}_{1}=2 a b, \quad \sqrt{d_{1}} \tilde{y}_{2}=a^{2}-b^{2},
$$

where $a$ and $b$ are coprime. Then we can write the second identity in (6) as

$$
\begin{aligned}
\tilde{y}_{1}^{2}+d_{2} \tilde{y}_{2}^{2}=\tilde{y}_{1}^{2}+\left(\frac{\sqrt{d_{2}}}{\sqrt{d_{1}}}\left(\sqrt{d_{1}} \tilde{y}_{2}\right)\right)^{2} & =4 a^{2} b^{2}+\frac{d_{2}}{d_{1}}\left(a^{2}-b^{2}\right)^{2} \\
& =\left(\frac{d_{2}}{d_{1}}-1\right)\left(a^{2}-b^{2}\right)+\left(a^{2}+b^{2}\right) \\
& =\left(\sqrt{\left(\frac{d_{2}}{d_{1}}-1\right)}\left(a^{2}-b^{2}\right)\right)^{2}+\left(a^{2}+b^{2}\right)^{2} .
\end{aligned}
$$


Since $d_{2} \neq d_{1}$ we have that setting $\gamma=\sqrt{\left(\frac{d_{2}}{d_{1}}-1\right)}$ then $\gamma \neq 0$. Let

$$
a_{1}=\sqrt{\gamma} a, \quad b_{1}=\sqrt{\gamma} b .
$$

Then

$\tilde{y}_{1}^{2}+d_{2} \tilde{y}_{2}^{2}=\left(\gamma\left(a^{2}-b^{2}\right)\right)^{2}+\left(a^{2}+b^{2}\right)^{2}=\left(a_{1}^{2}-b_{1}^{2}\right)^{2}+\gamma^{-2}\left(a_{1}^{2}+b_{1}^{2}\right)^{2}=Y_{1}^{2}+X_{1}^{2}$.

In view of Lemma 4 since $Y_{1}=a_{1}^{2}-b_{1}^{2}$ we must have that $X_{1}=2 a_{1} b_{1}$. Therefore

$$
X_{1}=\gamma^{-1}\left(a_{1}^{2}+b_{1}^{2}\right)=2 a_{1} b_{1}, \quad \text { that is } \quad a_{1}^{2}+b_{1}^{2}-2 \gamma a_{1} b_{1}=0 .
$$

This yields

$$
a_{1}=\gamma b_{1} \pm b_{1} \sqrt{\gamma^{2}-1}=b_{1}\left(\gamma \pm \sqrt{\gamma^{2}-1}\right)
$$

Since $\gamma \pm \sqrt{\gamma^{2}-1} \neq 0$ and $a$ and $b$ are coprime (and so are $a_{1}$ and $b_{1}$ ) we get a contradiction. This proves the claim.

In short, there is at most one constant $c \notin\{0,1\}$ such that $c \tilde{y}_{1}^{2}+(1-c) \tilde{y}_{2}^{2}$ is a square of a polynomial meaning that equation (2) has at most seven different polynomial solutions $0, \pm y_{1}, \pm y_{2}$ and $y_{0}$ as in (5).

Example 1. We consider the equivariant polynomial Abel differential equation (2) with

$$
\begin{aligned}
a(x) & =-2 x+48 x^{3}-768 x^{7}+512 x^{9}, \\
b_{1}(x) & =2\left(-1+96 x^{4}-1536 x^{6}+768 x^{8}\right), \\
b_{3}(x) & =64 .
\end{aligned}
$$

This equation has the following 7 polynomial solutions

$$
\begin{aligned}
y_{1}(x) & =0, \\
y_{2,3}(x) & = \pm\left(2 \sqrt{2} x^{3}+\frac{x}{\sqrt{2}}\right), \\
y_{4,5}(x) & = \pm\left(x-4 x^{3}\right), \\
y_{6,7}(x) & = \pm\left(\frac{1}{4 \sqrt{2}}-2 \sqrt{2} x^{4}\right) .
\end{aligned}
$$

\section{Proof of Theorem 2}

First we recall that if $y(x) \neq 0$ is a solution of equation (4), then $-y(x)$ is also a solution of equation (4) which is different from $y(x)$.

Lemma 5. Let $y_{0}(x) \neq 0, y_{1}(x), y_{2}(x)$ be polynomial solutions of equation (4) such that $y_{1}(x) \not \equiv 0, y_{2}(x) \not \equiv 0$ and $y_{2}(x) \neq-y_{1}(x)$. Set $y_{1}(x)=$ $g(x) \tilde{y}_{1}(x)$ and $y_{2}(x)=g(x) \tilde{y}_{2}(x)$ where $g=\operatorname{gcd}\left(y_{1}, y_{2}\right)$. Then, except the solution $y=0$, all the other polynomial solutions of equation (4) can be expressed as

$$
y_{0}(x ; c)= \pm g(x)\left(c \tilde{y}_{1}^{2}(x)+(1-c) \tilde{y}_{2}^{2}(x)\right)^{1 / 2},
$$


where $c$ is a constant.

Proof. Let $y$ be a nonzero polynomial solution of equation (2). The functions $z_{0}=y_{0}^{2}, z_{1}=y_{1}^{2}$ and $z_{2}=y_{2}^{2}$ are solutions of a linear differential equation and satisfy

$$
a(x) \dot{z}_{i}=2 b_{0}(x)+2 b_{2}(x) z_{i}, \quad i=0,1,2 .
$$

Therefore we have

$$
\frac{\dot{z}_{0}(x)-\dot{z}_{1}(x)}{z_{0}(x)-z_{1}(x)}=\frac{\dot{z}_{2}(x)-\dot{z}_{1}(x)}{z_{2}(x)-z_{1}(x)} .
$$

Integrating this equality we obtain

$$
z_{0}(x)=z_{1}(x)+c\left(z_{2}(x)-z_{1}(x)\right),
$$

with $c$ an arbitrary constant. So the general solution of equation (2) is

$$
\begin{aligned}
y_{0}^{2}(x) & =z_{0}(x)=z_{1}(x)+c\left(z_{2}(x)-z_{1}(x)\right) \\
& =(1-c) y_{1}^{2}(x)+c y_{2}^{2}(x)=g^{2}(x)\left((1-c) \tilde{y}_{1}^{2}+c \tilde{y}_{2}^{2}\right),
\end{aligned}
$$

with $c$ an arbitrary constant.

In view of Lemma 3 , if $y_{1}(x), y_{2}(x)$ are polynomial solutions of equation (4) such that $y_{1}(x) \not \equiv 0, y_{2}(x) \not \equiv 0$ and $y_{2}(x) \neq-y_{1}(x)$ then any other polynomial solution is of the form as in (7) for some appropriate constant $c$. In particular, $c \tilde{y}_{1}^{2}(x)+(1-c) \tilde{y}_{2}^{2}(x)$ is a square of a polynomial $P$. Proceeding exactly as in the proof of Theorem 1 we conclude that equation (4) has at most seven different polynomial solutions $0, \pm y_{1}, \pm y_{2}$ and $y_{0}$ as in (7).

Example 2. We consider the equivariant polynomial Abel differential equation of second kind (4) with

$$
\begin{aligned}
a(x) & =2 x^{4}-3 x^{2}+\frac{1}{8}, \\
b_{0}(x) & =\frac{x}{2}-8 x^{5}, \\
b_{2}(x) & =4 x^{3}-3 x .
\end{aligned}
$$

This equation has the following 7 polynomial solutions

$$
\begin{aligned}
y_{1}(x) & =0 \\
y_{2,3}(x) & = \pm\left(2 x^{2}-\frac{1}{2}\right) \\
y_{4,5}(x) & = \pm\left(\sqrt{2} x^{2}+\frac{1}{2 \sqrt{2}}\right), \\
y_{6,7}(x) & = \pm 2 x
\end{aligned}
$$




\section{ACKNowledGements}

The first author is partially supported by a FEDER-MINECO grant MTM2016-77278-P, a MINECO grant MTM2013-40998-P, and an AGAUR grant number 2014SGR-568. The second author is partially supported by FCT/Portugal through UID/MAT/04459/2013.

\section{REFERENCES}

[1] D. Behloul, S.S. Cheng, Computation of all polynomial solutions of a class of nonlinear differential equations, Computing 77 (2006), 163-177.

[2] M. Briskin, J.P. Françoise, Y. Yomdin, Center conditions, compositions of polynomials and moments on algebraic curves, Ergodic Theory \& Dynam. Systems 19 (1999), 1201-1220.

[3] M. Briskin, J.P. Françoise, Y. Yomdin, Center conditions. II. Parametric and model center problems, Israel J. Math. 118 (2000), 61-82.

[4] M. Briskin, J.P. Françoise, Y. Yomdin, Center conditions. III. Parametric and model center problems, Israel J. Math. 118 (2000), 83-108.

[5] J.G. Campbell, M. Golomb, On the polynomial solutions of a Riccati equation, The Amer. Math. Monthly 61 (1954), 402-404.

[6] A. Ferragut And J. Llibre, On the polynomial solutions of the polynomial differential equations $y \dot{y}=a_{0}(x)+a_{1}(x) y^{2}+\ldots+a_{n}(x) y^{n}$, Preprint, 2016.

[7] A. Gasull, J. Llibre, Limit cycles for a class of Abel equations, SIAM J. Math. Anal. 21 (1990), 1235-1244.

[8] A. Gasull, J. Torregrosa And X. Zhang The number of polynomial solutions of polynomial Riccati equations, J. Differential Equations 261 (2016), 5071-5093.

[9] J. Giné, M. Grau And J. Llibre, On the polynomial limit cycles of polynomial differential equations, Israel J. Math. 181 (2011), 461-475.

[10] E. Kamke, Differentialgleichungen: Lösungsmethoden und Lösungen, Springer 1977.

[11] A. Lins Neto, On the number of solutions of the equation $d x / d t=\sum_{j=0}^{n} a_{j}(t) x^{j}$, $0 \leq t \leq 1$, for which $x(0)=x(1)$, Invent. Math. 59 (1980), 67-76.

[12] M.P. MARKAKIS, Closed-form solutions of certain Abel equations of the first kind, Appl. Math. Lett. 22 (2009), 1401-1405.

[13] D.E. Panayotounakos, Exact analytic solutions of unsolvable classes of first and second order nonlinear ODEs. I. Abel's equations, Appl. Math. Lett. 18 (2005), 155162.

[14] E.D. RAinville, Necessary conditions for polynomial solutions of certain Riccati equations, The Amer. Math. Monthly 43 (1936), 473-476.

2 Departament de Matemàtiques, Universitat Autònoma de Barcelona, 08193 Bellaterra, Barcelona, Catalonia, Spain

E-mail address: jllibre@mat.uab.cat

${ }^{3}$ Departamento de Matemática, Instituto Superior Técnico, Universidade Técnica de Lisboa, Av. Rovisco Pais 1049-001, Lisboa, Portugal

E-mail address: cvalls@math.ist.utl.pt 